\title{
Changes of Antimicrobial Resistance Causing Infections Following Transrectal Prostate Biopsy: Analysis of 10-Year Data
}

\author{
Da Eun Han, Sun Tae Ahn, Jong Wook Kim, Du Geon Moon, Hong Seok Park, Mi Mi Oh \\ Department of Urology, Korea University College of Medicine, Seoul, Korea
}

Purpose: The production of extended-spectrum $\beta$-lactamases (ESBLs) has emerged as one of the main causes of antimicrobial resistance. It is well known that infections of ESBL-producing Enterobacteriaceae causes poor clinical outcomes. This study investigated the changes in the antimicrobial resistance patterns in infections following transrectal ultrasound-guided prostate (TR) biopsy over a 10 year period and analyzed whether the clinical course varies in infections caused by the ESBL-producing Enterobacteriaceae.

Materials and Methods: We retrospectively analyzed patients who had infections after a TR biopsy at the Korea University Guro Hospital from January 2010 to October 2019. Infection from a TR biopsy was defined as readmission due to a fever of 38 degrees or higher that occurred within one week after the biopsy.

Results: Among 1,855 patients who received a TR biopsy, 39 patients (2.10\%) had infectious complications. Of 33 culture-positive patients, 29 patients (87.9\%) showed quinolone resistance, 10 patients (30.3\%) were ESBL-positive and 9 patients had concomitant quinolone resistance and were also ESBL-positive. 75\% of ESBL-positive bacterial infections occurred after 2016 indicating increasing incidence in recent days. The only significant difference in the clinical course between the ESBL-negative and the positive group was the lower systolic blood pressure of the ESBL-positive group during hospitalization ( $p$-value $=0.018$ ).

Conclusions: Infections due to the ESBL-producing Enterobacteriaceae showed a tendency to increase among TR biopsy patients since 2016. Although the clinical course of the ESBL-positive infection did not show significant differences to ESBL-negative infection, further analysis is needed because of the small number of patients.

Keywords: Prostate; Biopsy; Transrectal; Infections; Beta-lactam resistance

Copyright $($ ) 2021, Korean Association of Urogenital Tract Infection and Inflammation.

(c) (1) (s) This is an open access article distributed under the terms of the Creative Commons Attribution (C) (1) (\$) Non-Commercial License (http://creativecommons.org/licenses/by-nc/4.0) which permits unrestricted non-commercial use, distribution, and reproduction in any medium, provided the original work is properly cited.
Received: 27 October, 2021

Revised: 10 December, 2021

Accepted: 16 December, 2021

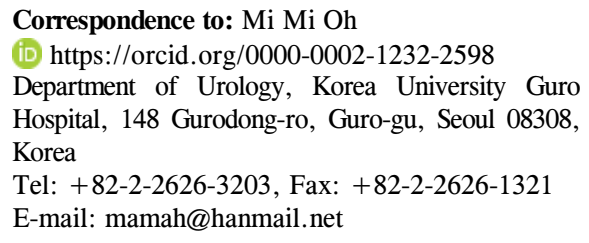

\section{INTRODUCTION}

As more people undergo a prostate-specific antigen (PSA) test for health checkups, the number of prostate biopsies is also rising. The transrectal ultrasound-guided prostate (TR) biopsy is used most frequently to detect prostate cancer [1].
In addition, the prostate biopsy-related infection rate has increased from $0.1 \%$ to $7.0 \%$. Among them, the sepsis rate has increased from $0.3 \%$ to $3.1 \%$ [2,3]. A possible explanation for this trend is the high incidence of antibiotic resistance in the community. In Korea, the fluoroquinolone resistance of Escherichia coli has reached $48 \%$. Following quinolone 
resistance (QR), extended-spectrum $\beta$-lactamase (ESBL)producing Enterobacteriaceae has emerged as one of the leading causes of antimicrobial resistance. Approximately a quarter of Enterobacteriaceae had ESBL in Korea [4,5]. In addition, ESBL-producing Enterobacteriaceae causes the worse clinical outcome [5,6].

This study investigated the changes in antimicrobial resistance of infectious complications following a TR biopsy in 10 years, and analyzed whether the clinical course varies according to the infections caused by ESBL-producing Enterobacteriaceae.

\section{MATERIALS AND METHODS}

Patients who had infectious complications after a TR biopsy in Korea University Guro Hospital from January 2010 to October 2019 were analyzed retrospectively. The patients were selected for the biopsy if they had one or more of the following criteria: palpable nodules or abnormal signs on a digital rectal examination, PSA level higher than $4 \mathrm{ng} / \mathrm{ml}$ with no pyuria, and hypoechoic lesion in transrectal ultrasound. Those with a PSA level higher than $2 \mathrm{ng} / \mathrm{ml}$ were included if the patient had been taking 5-alpha reductase inhibitors for more than six months.

All biopsies were performed by a TR, 12-core systematic biopsy using the same biopsy gun product. A rectal povidone-iodine preparation after an enema was performed prior to the biopsy. Regarding prophylactic antibiotics, intravenous ciprofloxacin $400 \mathrm{mg}$ was administered just before and within 24 hours from the biopsy. After discharge, oral fosfomycin $3 \mathrm{~g}$ was administered twice per day.

The infection due to the TR biopsy was defined as prolonged hospitalization or readmission due to a fever of $38^{\circ} \mathrm{C}$ or higher, accompanied by low urinary tract symptoms and pyuria that occurred within one week after the biopsy. Stable vital signs lasting more than two days and normalized laboratory results of inflammation were required before the patient was discharged.

The following patient data were retrieved: age, PSA, prostate volume, medical comorbidity, history of antibiotics usage, and Foley catheter insertion three months before the procedure, whether other surgeries were performed at the time of the biopsy, bacterial strain, the susceptibility to antibiotics of a urine and blood culture, and the types of antibiotics used to treat fever. The changes in antimicrobial resistance, particularly QR and the presence of ESBL-producing over 10 years, were assessed.

The presence of sepsis, highest fever, lowest systolic blood pressure (SBP), highest white blood cell count (WBC), erythrocyte sedimentation rate (ESR), and C-reactive protein (CRP), fever duration, total admission days were reviewed to characterize the clinical course of infection. Sepsis was defined as a confirmed or suspected infection with systemic inflammatory response syndrome (SIRS). SIRS requires two or more of following four signs: body temperature $>38^{\circ} \mathrm{C}$ or $<36{ }^{\circ} \mathrm{C}$, heart rate $>90 / \mathrm{min}$, respiratory rate $>20 / \mathrm{min}$ or $\mathrm{PaCO}_{2}<32 \mathrm{mmHg}$ and $\mathrm{WBC}>12 \times 10^{3} / \mu \mathrm{l}$ or $<4 \times 10^{3} / \mu \mathrm{l}$ or $>10 \%$ immature band neutrophils of total WBC [7]. The difference in the baseline and clinical course characteristics between the ESBL-negative and the positive infections were analyzed.

Table 1. Baseline characteristics of the patients with infectious complications $(n=39)$

\begin{tabular}{lc}
\multicolumn{1}{c}{ Variable } & Value \\
\hline Age $(\mathrm{yr})$ & $63 \pm 9.62$ \\
PSA $(\mathrm{ng} / \mathrm{ml})$ & $7.63 \pm 3.71$ \\
Prostate volume $(\mathrm{g})$ & $46.65 \pm 19.12$ \\
Prostate cancer & $6(15.4)$ \\
HTN & $19(48.7)$ \\
CHF & $10(25.6)$ \\
COPD & $3(7.7)$ \\
CKD & $3(7.7)$ \\
Liver disease & $3(7.7)$ \\
DM & $16(41.0)$ \\
Neurologic Dz & $8(20.5)$ \\
BPH medication & $34(87.2)$ \\
h/o Foley catheter insertion & $4(10.3)$ \\
Concomittant op & $7(17.9)$ \\
Usage of immunosuppressants & $2(5.1)$ \\
h/o Cephalosporin usage & $4(10.3)$ \\
h/o Fluoroquinolone usage & $7(17.9)$ \\
Antibiotics treatment & \\
Ertapenem & $21(53.8)$ \\
Cefotaxime & $3(7.7)$ \\
Ciprofloxacin & $7(17.9)$ \\
Amikacin+Ciprofloxacin & $1(2.6)$ \\
Ceftriaxone & $1(2.6)$ \\
Piperacillin/tazobactam & $1(2.6)$ \\
Meropenem & $4(10.3)$ \\
Cefpiramide & $1(2.6)$ \\
\hline
\end{tabular}

Values are presented as mean \pm standard deviation or number (\%). Concomitant op means that TR biopsy was done during urologic surgery.

PSA: prostate-specific antigen, HTN: hypertension, CHF: congestive heart failure, COPD: chronic obstructive pulmonary disease, CKD: chronic kidney disease, DM: diabetes mellitus, Neurologic Dz: neurologic diseases, such as a past cerebrovascular accident or spinal cord injury, BPH: benign prostate hyperplasia, h/o: history of, TR: transrectal ultrasound-guided prostate. 


\section{Statistical Analyses and Ethical Statement}

A Mann-Whitney U-test and Fisher's exact test were used to analyze the continuous and categorical variables, respectively. All statistical analyses were performed using IBM SPSS version 24.0 (IBM Co., Armonk, NY, USA). All calculated p-values were two-sided, with statistical significance defined as $\mathrm{p}<0.05$. This study proposal was approved by the Korea University Guro Hospital Institutional Review Board (IRB No. 2021GR0456). The demand for participants' informed consent was waived by the IRB.

\section{RESULTS}

From January 2010 to October 2019, 1,855 TR biopsies were performed. Among them, 39 patients (2.10\%) had infectious complications and required admission. Table 1 lists the baseline characteristics. Ertapenem was the most commonly used empirical antibiotic for infection complications, followed in order by ciprofloxacin and meropenem (Table 1).

\section{A}

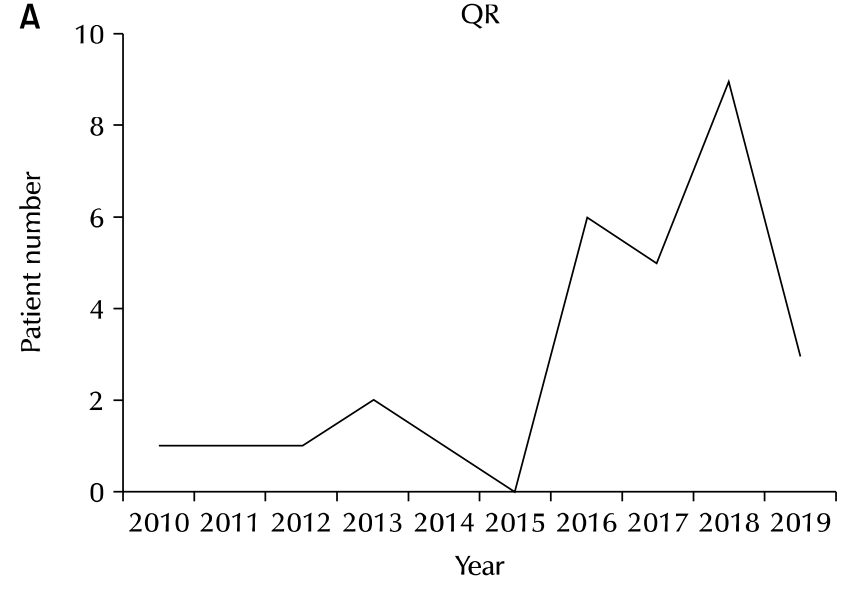

C

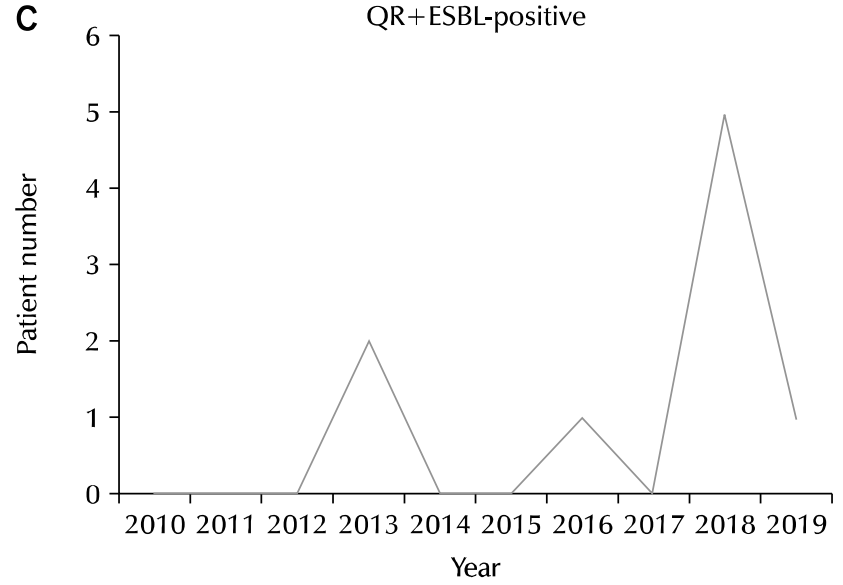

All patients underwent culture tests from the blood and urine on the readmission date. Bacteria were identified in 33 patients: 19 in both urine and blood, eight in the blood only, and six in the urine only. E. coli was identified in all patients except for one in which Staphylococcus aureus was identified. Of the 33 culture-positive patients, 29 (87.9\%), 10 (30.3\%), and nine (27.3) patients showed QR, ESBL-positive, and concomitant QR plus ESBL-positive, respectively. During the study period, QR bacteria occurred in all periods except at 2015 and showed a sharp increase after 2016 (Fig. 1A). ESBL-positive bacteria were identified intermittently, but $75 \%$ of them occurred after 2016 (Fig. 1B). The incidence of both QR and ESBL-positive bacteria increased their incidence over time (Fig. 1C).

There was no significant difference in the basic characteristics of patients with or without ESBL production. On the other hand, patients with ESBL-producing bacteria had a lower SBP and higher ESR values than those without ESBL-producing bacteria (Table 2).

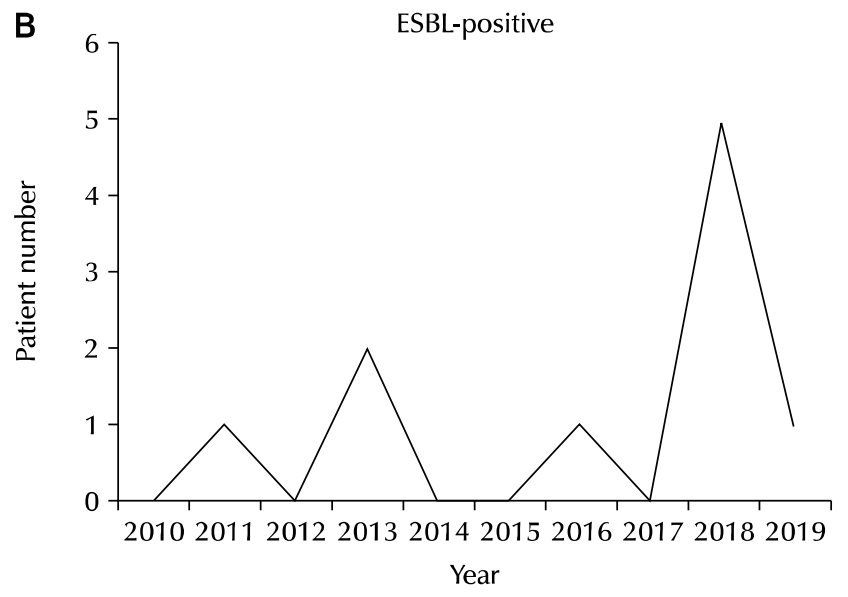

Fig. 1. (A-C) Incidence of antibiotic resistance infection by year. (A) Quinolone resistance $(\mathrm{QR})$ incidence. (B) Extended-spectrum $\beta$-lactamase (ESBL) production incidence. (C) Concomitant QR and ESBL production. 
Table 2. Difference in the clinical course and baseline characteristics between ESBL negative and positive infection

\begin{tabular}{|c|c|c|c|}
\hline Variable & $\operatorname{ESBL}(-), n=22$ & $\operatorname{ESBL}(+), n=10$ & $\mathrm{p}$-value \\
\hline Age $(y r)$ & $63.8 \pm 10.6$ & $60.3 \pm 6.6$ & 0.25 \\
\hline PSA (ng/ml) & $8.0 \pm 4.3$ & $7.67 \pm 2.9$ & 0.705 \\
\hline Prostate volume (g) & $44.6 \pm 14.0$ & $46.0 \pm 16.6$ & 0.791 \\
\hline Prostate cancer & $10(45.5)$ & $2(20.0)$ & 0.228 \\
\hline HTN & $11(50.0)$ & $5(50.0)$ & $>0.999$ \\
\hline $\mathrm{CHF}$ & $6(27.3)$ & $3(30.0)$ & $>0.999$ \\
\hline COPD & $2(9.1)$ & $1(10.0)$ & $>0.999$ \\
\hline CKD & $2(9.1)$ & $1(10.0)$ & $>0.999$ \\
\hline Liver disease & $3(13.6)$ & 0 & 0.539 \\
\hline DM & $9(40.9)$ & $3(30.0)$ & $>0.999$ \\
\hline Neurologic Dz & $6(27.3)$ & $1(10.0)$ & 0.644 \\
\hline BPH medication & $22(100)$ & $8(80.0)$ & 0.201 \\
\hline h/o Foley catheter insertion & $2(9.1)$ & $1(10.0)$ & $>0.999$ \\
\hline Concomittant op & $5(22.7)$ & 0 & 0.291 \\
\hline Usage of immnosupressant & $2(9.1)$ & 0 & $>0.999$ \\
\hline h/o Cephalosporin usage & $2(9.1)$ & $1(10.0)$ & $>0.999$ \\
\hline h/o Fluoroquinolone usage & $3(13.6)$ & $4(40.0)$ & 0.157 \\
\hline Post biopsy sepsis & $18(75.0)$ & $9(90.0)$ & 0.644 \\
\hline Highest fever $\left({ }^{\circ} \mathrm{C}\right)$ & $39.0 \pm 0.79$ & $39 \pm 0.75$ & 0.88 \\
\hline Lowest SBP (mmHg) & $101.9 \pm 12.37$ & $89.7 \pm 12.52$ & 0.023 \\
\hline Highest WBC $(* 10 \mathrm{C} \mu \mathrm{l})$ & $10.9 \pm 220.1$ & $15.2 \pm 8421.1$ & 0.212 \\
\hline Highest ESR $(\mathrm{mm} / \mathrm{hr})$ & $40 \pm 35.4$ & $49.6 \pm 30.2$ & 0.045 \\
\hline Highest CRP (mg/dl) & $142.0 \pm 81.1$ & $180.5 \pm 77.0$ & 0.174 \\
\hline Fever duration (days) & $6.3 \pm 7.4$ & $5.80 \pm 3.4$ & 0.541 \\
\hline Total adm duration (days) & $10.7 \pm 7.5$ & $9.80 \pm 1.9$ & 0.328 \\
\hline
\end{tabular}

Values are presented as mean \pm standard deviation or number (\%).

Concomitant op means that TR biopsy was done during urologic surgery.

ESBL: extended-spectrum $\beta$-lactamase, PSA: prostate-specific antigen, HTN: hypertension, CHF: congestive heart failure, COPD: chronic obstructive pulmonary disease, CKD: chronic kidney disease, DM: diabetes mellitus, Neurologic Dz: neurologic diseases, such as a past cerebrovascular accident or spinal cord injury, BPH: benign prostate hyperplasia, h/o: history of, SBP: systolic blood pressure, WBC: white blood cell count, ESR: erythrocyte sedimentation rate, CRP: C-reactive protein, adm: hospital admission, TR: transrectal ultrasound-guided prostate.

\section{DISCUSSION}

Observational studies at a single center from 2010 to 2019 revealed a $2.10 \%$ readmission rate due to infection complications after TR biopsy. Bacteria were cultured in 33 patients with E. coli accounted for 97.0\%. QR was $87.9 \%$, and the ESBL-positive rate was 30.3\%. From 2016, a sharp increase in QR was noted, and 79.3\% occurred after 2016. The ESBL-positive cases also mostly appeared after 2016, comprising $75 \%$ of the total incidence. All ESBL-positive bacteria except for one that occurred in 2011 showed QR. The number of bacteria showing both QR and ESBL-positive also increased with time, and $77.8 \%$ were identified after 2016. According to a review article conducted in Korea, the post-TR biopsy incidence ranged from $0.65 \%$ to $3.1 \%$ [1]. A multicenter study including Korea revealed a 3.1\% readmission rate due to a post-TR biopsy infection [8]. Another study conducted in Korea from 1995 to 2013 found that $95.0 \%$ of post-TR biopsy infections were caused by $E$. coil. The QR and ESBL-positive rates were $93.6 \%$ and $21.3 \%$, respectively, and all ESBL-positive bacteria showed QR [9].
After reviewing these references, the infection rate at the authors' institution was not inferior to other centers and had similar outcomes regarding the bacterial species and their resistance rate.

Many studies claimed that ESBL-producing Enterobacteriaceae infections have a more devastating clinical course. First, it is more virulent than the ESBL-negative strain. Second, the use of antibiotics that target ESBL-positive bacteria is delayed $[6,10]$. In a preemptive study conducted at the same center as in this investigation, post-TR biopsy ESBL-positive infections had a longer fever duration, hospital days, and higher infection serum markers than a negative infection [6]. On the other hand, there was no clinical difference in this study except for the lowest SBP. This can be explained by the use of carbapenem as empirical antibiotics in $64.1 \%$ of cases, considering that the causative bacteria of fever are ESBL-positive. Many studies recommend different empirical antibiotics for post-TR biopsy infection, but the central concept is identical. Broad-spectrum antibiotics covering both QR and ESBL-positive bacteria, such as carbapenem, amikacin, or third-generation 
cephalosporin, are used initially [1,2,9]. As the carbapenem resistance rate is relatively lower than cephalosporin in Korea, the institution administered carbapenem first for post-TR biopsy infections [4].

There were some limitations to this study. First, it was a retrospective study, and the patient's medical history, particularly prior biopsy antibiotics usage, may not be accurate. Second, statistical significance was difficult to derive because of the small number of infections. Furthermore, the risk factors for ESBL-positive infection could not be analyzed.

Nevertheless, there was a change in the antibiotic resistance rate of patients with complications of infection after TR biospy over the recent 10 years. In particular, the increasing trend of ESBL-positive strain was confirmed.

\section{CONCLUSIONS}

Infectious complications among TR biopsy patients due to ESBL-producing Enterobacteriaceae have shown a tendency to increase since 2016. There were no significant differences in the clinical courses based on the result of the ESBL-producing Enterobacteriaceae. Further analysis will be needed because of the small number of patients.

\section{CONFLICT OF INTEREST}

No potential conflict of interest relevant to this article was reported.

\section{ACKNOWLEDGMENTS}

This work was supported by a KAUTII research grant.

\section{AUTHOR CONTRIBUTIONS}

D.E.H. participated in data collection and wrote the manuscript. M.M.O., S.T.A. and D.E.H. participated in the study design and performed the statistical analysis. J.W.K., D.G.M., and H.S.P. participated in the study design and coordination and helped to draft the manuscript. All authors read and approved the final manuscript.

\section{ORCID}

Da Eun Han, https://orcid.org/0000-0002-6395-5651

Sun Tae Ahn, https://orcid.org/0000-0003-1233-5951

Jong Wook Kim, https://orcid.org/0000-0003-2228-0640

Du Geon Moon, https://orcid.org/0000-0002-9031-9845

Hong Seok Park, https://orcid.org/0000-0001-7287-2682

Mi Mi Oh, https://orcid.org/0000-0002-1232-2598

\section{REFERENCES}

1. Lee SJ. Infection after transrectal ultrasound-guided prostate biopsy. Korean J Urol 2015;56:346-50.

2. Liss MA, Ehdaie B, Loeb S, Meng MV, Raman JD, Spears V, et al. An update of the American Urological Association white paper on the prevention and treatment of the more common complications related to prostate biopsy. J Urol 2017;198: 329-34.

3. Nam RK, Saskin R, Lee Y, Liu Y, Law C, Klotz LH, et al. Increasing hospital admission rates for urological complications after transrectal ultrasound guided prostate biopsy. J Urol 2010;183:963-8.

4. Kim D, Ahn JY, Lee $\mathrm{CH}$, Jang SJ, Lee $\mathrm{H}$, Yong D, et al. Increasing resistance to extended-spectrum cephalosporins, fluoroquinolone, and carbapenem in gram-negative bacilli and the emergence of carbapenem non-susceptibility in klebsiella pneumoniae: analysis of Korean Antimicrobial Resistance Monitoring System (KARMS) data from 2013 to 2015. Ann Lab Med 2017;37:231-9.

5. Rodriguez-Bano J, Picon E, Gijon P, Hernandez JR, Ruiz M, Pena C, et al.; Spanish Network for Research in Infectious Diseases (REIPI). Community-onset bacteremia due to extended-spectrum beta-lactamase-producing Escherichia coli: risk factors and prognosis. Clin Infect Dis 2010;50:40-8.

6. Oh MM, Chae JY, Kim JW, Kim JW, Yoon CY, Park MG, et al. Positive culture for extended-spectrum $\beta$-lactamase during acute prostatitis after prostate biopsy is a risk factor for progression to chronic prostatitis. Urology 2013;81:1209-12.

7. American College of Chest Physicians/Society of Critical Care Medicine Consensus Conference: definitions for sepsis and organ failure and guidelines for the use of innovative therapies in sepsis. Crit Care Med 1992;20:864-74.

8. Wagenlehner FM, van Oostrum E, Tenke P, Tandogdu Z, Cek M, Grabe $M$, et al.; GPIU investigators. Infective complications after prostate biopsy: outcome of the Global Prevalence Study of Infections in Urology (GPIU) 2010 and 2011, a prospective multinational multicentre prostate biopsy study. Eur Urol 2013;63:521-7.

9. Song W, Choo $\mathrm{SH}$, Sung $\mathrm{HH}$, Han $\mathrm{DH}$, Jeong BC, Seo SI, et al. Incidence and management of extended-spectrum beta-lacta- 
$60 \mathrm{Da}$ Eun Han, et al. Antibiotic Resistance of Post Prostate Biopsy Infection

mase and quinolone-resistant Escherichia coli infections after prostate biopsy. Urology 2014;84:1001-7.

10. Melzer M, Petersen I. Mortality following bacteraemic infection caused by extended spectrum beta-lactamase (ESBL) producing E. coli compared to non-ESBL producing E. coli. J Infect 2007;55:254-9. 